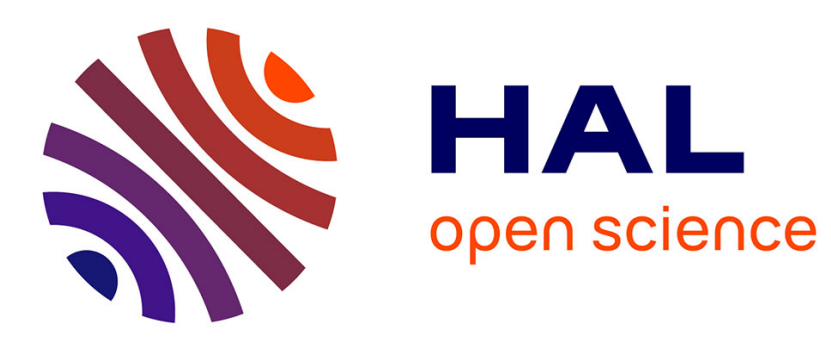

\title{
Evaluation of a new optimisation algorithm for rigid registration of MRI data
}

\author{
Nicolas Wiest-Daesslé, Pierre Yger, Sylvain Prima, Christian Barillot
}

\section{To cite this version:}

Nicolas Wiest-Daesslé, Pierre Yger, Sylvain Prima, Christian Barillot. Evaluation of a new optimisation algorithm for rigid registration of MRI data. Medical Imaging 2007: Image Processing, Feb 2007, San Diego, United States. 10.1117/12.707931 . inria-00133375

\section{HAL Id: inria-00133375 https://hal.inria.fr/inria-00133375}

Submitted on 26 Feb 2007

HAL is a multi-disciplinary open access archive for the deposit and dissemination of scientific research documents, whether they are published or not. The documents may come from teaching and research institutions in France or abroad, or from public or private research centers.
L'archive ouverte pluridisciplinaire HAL, est destinée au dépôt et à la diffusion de documents scientifiques de niveau recherche, publiés ou non, émanant des établissements d'enseignement et de recherche français ou étrangers, des laboratoires publics ou privés. 


\title{
Evaluation of a new optimisation algorithm for rigid registration of MRI data
}

\author{
Nicolas Wiest-Daesslé ${ }^{a, b, c, d}$, Pierre Yger ${ }^{a, b, c, d, e}$, Sylvain Prima ${ }^{a, b, c, d}$ and Christian \\ Barillot ${ }^{a, b, c, d}$ \\ ${ }^{a}$ INRIA, VisAGeS U746 Unit/Project, IRISA, Campus de Beaulieu, F-35042 Rennes, France \\ ${ }^{b}$ University of Rennes I, IRISA, Campus de Beaulieu, F-35042 Rennes, France \\ ${ }^{c}$ CNRS, IRISA UMR 6074, Campus de Beaulieu, F-35042 Rennes, France \\ ${ }^{d}$ INSERM, VisAGeS U746 Unit/Project, IRISA, Campus de Beaulieu, F-35042 Rennes, France \\ ${ }^{e}$ ENS Cachan, Brittany Extension - CS/IT Department, F-35170 Bruz, France
}

\begin{abstract}
We propose to use a recently introduced optimisation method in the context of rigid registration of medical images. This optimisation method, introduced by Powell and called NEWUOA, is compared with two other widely used algorithms: Powell's direction set and Nelder-Mead's downhill simplex method. This paper performs a comparative evaluation of the performances of these algorithms to optimise different image similarity measures for different mono- and multi-modal registrations. Images from the BrainWeb project are used as a gold standard for validation purposes. This paper exhibits that the proposed optimisation algorithm is more robust, more accurate and faster than the two other methods.
\end{abstract}

\section{INTRODUCTION}

Longitudinal and cross-sectional medical imaging studies require good images alignment for voxelwise comparison of mono- and multi-modal data. Multiple registration methods have been developed over the last twenty years. A popular approach is to optimise a similarity measure between the intensities of the images to register. Many similarity measures and many optimisation methods have been proposed in the literature. ${ }^{1}$ In the present paper, a new method is introduced for the optimisation of different similarity measures in various multi-modal rigid registration contexts. This new algorithm, termed NEWUOA, was initially developed by Powell ${ }^{2}$ for large scale nonlinear optimisation problems where it was proved to be faster than standard techniques. NEWUOA is compared with two other widely used optimisation algorithms for image registration: Powell's direction set ${ }^{3}$ (POW) and Nelder-Mead's downhill simplex ${ }^{3}$ (SMP). Our goal is the determination of the best combination similarity measure / optimisation method for multi-modal rigid registration. Experiments are performed on synthetic data from the BrainWeb ${ }^{4}$ project. On average, mutual information optimised with NEWUOA is the most robust, most accurate and fastest method. Finally, results on real images are presented for other multimodal registration procedures (PET-to-MRI, SPECT-to-MRI).

\section{METHOD}

The rigid registration procedure can be addressed in an optimisation framework, in which the optimal transformation $\widehat{T}$ is defined as:

$$
\widehat{T}=\underset{T}{\arg \min } \Delta(I, T(J))
$$

where $I$ is a reference image, $J$ is a floating image, $T(J)$ is the image $J$ transformed using the transformation $T$, and $\Delta$ is a global similarity measure between the intensities of $I$ and $T(J)$ on their overlapping area. The transformation $T$ estimated here is a rigid-body transformation.

Further author information: Send correspondence to N. Wiest-Daesslé : nicolas.wiest-daessle@irisa.fr, Telephone: $+33299847120$ 
The current paper introduces a new optimisation method, NEWUOA, for rigid-body registration and focuses on the comparison of this method with SMP and POW. The comparison is performed here with four different similarity measures and multiple image modalities.

Section 2.1 gives an outline of the algorithm NEWUOA (for more details we refer to the original paper by Powell ${ }^{2}$ ). In Section 2.2, implementation details are discussed such as the definition of our rigid-body transformation, the details of the different similarity measures, and some specific details about our implementation for speed-up and robustness purposes.

\subsection{NEWUOA}

The image registration process, based on the intensities of the images such as the one described in Section 2, requires a system that allows the computation of the optimal parameters. In registration procedures, derivativefree optimisation methods are often employed for this task, mainly because, often, the analytical expression of the derivative of $\Delta$ does not exists. The computation of a discreet estimation of the derivative is a time consuming process. In the present paper, a recent derivative-free optimisation algorithm, called NEWUOA NEW Unconstrained Optimisation Algorithm) is employed for this task.

Modern optimisation algorithms often use a local model of the objective function $F$. According to Powell, ${ }^{5}$ the original idea is from Winfield (1973), ${ }^{6}$ who created a quadratic model $Q$ of the objective function $F$. In such case the model $Q$ is built to fit $F$, and the optimum of $Q$ is considered to match the one of $F$. With a non quadratic function, this is not true, so the model $Q$ is updated with the value of $F$ corresponding to the previous optimum of $Q$, and some iteration is performed until a stopping criterion is reached.

In 2000, Powell proposed an implementation of this approach termed Unconstrained Optimisation BY Quadratic Approximation (UOBYQA) ${ }^{5}$ which is the basis on which NEWUOA is constructed. The quadratic model $Q$ is created using $m$ sample points of $F$. A standard quadratic interpolation of a function requires $m=\frac{1}{2}(n+1)(n+2)$ samples for a problem of dimension $n$. A point $x_{0}$ is the initial parameter provided by the user; for our rigid registration case this is the identity transformation. Interpolation points are selected inside a neighborhood of $x_{0}$. This neighborhood is called the trust-region. The point $x_{n e w}$ is the optimal point computed inside the trust-region. The algorithm updates $Q$ by selecting the interpolation point $x_{\text {move }}$ that is the farthest from the point $x_{n e w}$. The point $x_{\text {move }}$ is replaced by $x_{\text {new }}$ and $Q$ is updated with the value of $F\left(x_{n e w}\right)$. The trust-region radius decreases during the optimisation process when the optimum of $Q$ stops decreasing the value of $F$. The iterations stop either when the trust-region radius reaches a user-defined final radius or when $Q$ is considered close enough to $F$.

NEWUOA uses the same method as UOBYQA, but one important speed factor of NEWUOA is the number of samples of the function $F$ used for the creation of $Q$. As stated before, the required number of interpolation point required for a perfect modeling of a quadratic model is exactly $\frac{1}{2}(n+1)(n+2)$. The initial construction of $Q$ is possible with less samples of $F$ thanks to additional constraints on the first and second derivatives of $Q$, refer to Section 3 of Powell ${ }^{2}$ for details. Reducing the number of calls of the objective function $F$ speeds up the algorithm which is especially interesting for rigid registration when this function $(\Delta)$ has a high computational burden. NEWUOA usually show good behaviour for optimisation when the number of samples is selected as $m=2 n+1$, which is the value used for the following experiments.

Finally the algorithm can be summarised as:

- Create an initial quadratic model $Q$ of the function to optimise $F$,

- Do the following iterations:

- Compute the minimum of $Q$ inside a trust-region,

- Improve the model using the latest optimum,

- Stop if the latest trust-region is lower than the user-defined end value,

- Stop if the distance between $Q$ and $F$ is small enough (perfect match of the model and the objective function $F$ ),

- Decrease the trust-region radius, if the values computed for $F$ stops decreasing. 


\subsection{Implementation of the registration algorithm}

\subsubsection{Transformation}

Image registration is seen here as an optimisation problem, where the searched transformation, $T$, maximises a similarity criterion, $\Delta$, between a reference image and a floating image deformed by $T$.

This paper only deals with three dimensional rigid transformations. Such a rigid transformation $T$ moves each point $p$ to $p^{\prime}$ following:

$$
p^{\prime}=T(p)=\mathbf{R}_{\mathbf{x}}\left(\phi_{x}\right) \mathbf{R}_{\mathbf{y}}\left(\phi_{y}\right) \mathbf{R}_{\mathbf{z}}\left(\phi_{z}\right) p+\mathbf{t}
$$

where $\mathbf{R}_{\mathbf{x}}$ (resp. $\mathbf{R}_{\mathbf{y}}$ and $\mathbf{R}_{\mathbf{z}}$ ) is a rotation of angle $\phi_{x}$ (resp. $\phi_{y}, \phi_{z}$ ) around the $x-($ resp. $y-$ and $z-$ ) axis and $\mathbf{t}=\left(t_{x}, t_{y}, t_{z}\right)$ is a translation.

\subsubsection{Similarity Measures}

This paper focuses on common state-of-the-art intensity-based similarity measures, such as the Correlation Coefficient $(\mathrm{CC})^{7}$ (suited for mono-modal cases), Mutual Information (MI), ${ }^{8}$ Normalised Mutual Information $(\mathrm{NMI})^{9}$ and Gradient-weighted Mutual Information (GMI) ${ }^{10}$ (suited for multi-modal cases).

To simplify the computation of each similarity measure, the measures are computed by means of a joint histogram of the images. The construction of the joint histogram is well described by Maes et al. ${ }^{8}$ In the following all those joint histograms are constructed using partial volume interpolation, this method being known as being one that reduces the interpolation artifacts that may arise during computation. ${ }^{8}$

\section{Correlation Coefficient}

The correlation coefficient (CC) is optimal when the dependency between the two images intensities is linear. This is a reasonable hypothesis in case of mono-modal image registration. This coefficient measures the dispersion of the joint intensities along a line. The correlation coefficient is mathematically described as follows:

$$
\rho=\frac{E\left(\left(I-\mu_{I}\right)\left(T(J)-\mu_{T(J)}\right)\right)}{\sigma_{I} \sigma_{T(J)}}
$$

where $I$ and $J$ are the intensities of the images, $T(J)$ is the image $J$ transformed by $T, \mu_{I}$ and $\mu_{T(J)}$ are the mean intensities of $I$ and $T(J)$, and $\sigma_{I}$ and $\sigma_{T(J)}$ are the standard deviations of $I$ and $T(J)$.

\section{Mutual Information}

The mutual information similarity measure is based on information theory and is related to the entropy of the joint histogram.

The computation, as defined by Maes et al. ${ }^{8}$ is based on the marginal and joint image intensity distributions $P(I), P(T(J))$ and $P(I, T(J)), I$ and $J$ being the images, $T(J)$ the image $J$ transformed by $T$ and can be written as follow:

$$
M I_{T}(I, J)=\sum_{I, J} P(I, T(J)) \log _{2} \frac{P(I, T(J))}{P(I) P(T(J))}
$$

\section{Normalised Mutual Information}

The normalised mutual information (NMI) is a symmetric and normalised version of the mutual information, defined as follows:

$$
N M I_{T}(I, J)=\frac{M I_{T}(I, J)}{H(I)+H(T(J))}
$$

where $H(I)$ and $H(T(J))$ are the marginal entropies of $I$ and $T(J)$. 


\section{Gradient-Weighted Mutual Information}

The Gradient-weighted mutual information (GMI) is a variation of the MI introduced by Pluim et al. ${ }^{10}$

The idea is to consider the information that may be contained in the gradients of the images to register in a way that will enhance the MI.

At each point $p$ of $I$ and its correspondant $p^{\prime}$ in $T(J)$, the angle $\alpha_{p, p^{\prime}}$ between the images intensity gradients $\nabla$ is computed as follows:

$$
\alpha_{p, p^{\prime}}=\operatorname{arcos} \frac{\nabla p \cdot \nabla p^{\prime}}{\|\nabla p\| \cdot\left\|\nabla p^{\prime}\right\|}
$$

During the registration process, to align the gradients in both images that have similar orientation (i.e an angle $\alpha_{p, p^{\prime}}$ near 0 or $\pi$ ), we consider a function of the angle given by:

$$
f(\alpha)=\frac{\cos (2 \alpha)+1}{2}
$$

Then, to avoid the difference of amplitude of such gradients that may be due to the intrisic nature of the images in case of a multimodal registration process, the orientation similarity is always weighted with the minimal amplitude of the gradients. Then, GMI can be expressed as follows:

$$
G M I_{T}(I, J)=G_{T}(I, J) M I_{T}(I, J)
$$

with

$$
G_{T}(I, J)=\sum_{\left(p, p^{\prime}\right) \in I \cap T(J)} \min \left(|\nabla p|,\left|\nabla p^{\prime}\right|\right) f\left(\alpha_{p, p^{\prime}}\right)
$$

\subsubsection{Optimisation}

In this paper, three optimisation algorithms are tested and compared, to estimate the six unknown parameters $\left(\phi_{x}, \phi_{y}, \phi_{z}, t_{x}, t_{y}\right.$ and $\left.t_{z}\right)$ : Powell's NEWUOA, ${ }^{2}$ Nelder-Mead's downhill simplex (SMP) ${ }^{3}$ and Powell's direction set $(\mathrm{POW})^{3}$ methods.

\subsubsection{Implementation details}

All similarity measures are computed from a joint histogram, with fixed number of bins $\left(256^{2}\right)$ and partial volume interpolation; points with corresponding coordinates outside the images grids are discarded from the computation. A multiresolution approach is chosen to increase the robustness, accuracy and to speed up the algorithm. A subsampled image is built by taking only one voxel out of $f_{x}$ (resp. $\left.f_{y}, f_{z}\right)$ in the $x$ - (resp. $y$-, $z$-) direction in the original image after Gaussian convolution with kernel size proportional to $f_{x}, f_{y}$ and $f_{z}$. A first solution is found at this low resolution, and then is used to initialise the algorithm at the higher resolution, and so on.

\section{RESULTS}

\subsection{BrainWeb MRI phantom data set}

For a good cross-evaluation of the capabilities of the optimisation algorithms NEWUOA, SMP and POW, in the context of multi-modal image registration, a ground truth data is built based on perfectly aligned Magnetic Resonance $(\mathrm{MR})$ images from the BrainWeb project $(181 \times 217 \times 181$ voxels). Multiple sequences are generated: T1-weighted, T2-weighted and Proton Density-weighted (PD). The spatial resolution for each volume is $1 \times 1 \times$ $1 \mathrm{~mm}^{3}$. All the BrainWeb images are corrupted with a $5 \%$ additive noise with respect to the highest intensity tissue. 


\subsection{Ground truth registration}

Three registration problems are considered: T1-to-T1, T2-to-T1, PD-to-T1. Fifty random transformations $T$ are generated with rotations $\left(\phi_{x}, \phi_{y}\right.$ and $\left.\phi_{z}\right)$ picked up uniformly in $\left[-5^{\circ},+5^{\circ}\right]$ and translations $\left(t_{x}, t_{y}\right.$ and $\left.t_{z}\right)$ also selected uniformly in $[-5 \mathrm{~mm},+5 \mathrm{~mm}]$ as shown in Fig. 1. The precision of the registration process is then quantified with a warping index $\omega$, as proposed by Thevenaz et al. ${ }^{11}$ The warping index is defined as follows: $J$ and $I$ are the BrainWeb MR images in perfect alignment. Two transformed images $I_{T}$ and $J_{T}$ are computed as follows: $I_{T}(x)=I(T(x))$ and $J_{T}(x)=J\left(T^{-1}(x)\right)$, where $T$ is a random transformation. The two transformations introduce similar interpolation artifacts in both images. The registration process shall recover $R=T \circ T$ which aligns $J_{T}$ with $I_{T}$. Let $\widehat{R}$ be the estimated transformation. The warping index $\omega$ measures the average geometric error between $R$ and $\widehat{R}$ :

$$
\omega=\frac{1}{\# \Omega\left(I_{T}\right)} \sum_{x \in \Omega\left(I_{T}\right)}\left\|R^{-1}(x)-\widehat{R}^{-1}(x)\right\|
$$

$\Omega\left(I_{T}\right)$ being the image grid of $I_{T}$ and $\|-\|$ the Euclidean norm.

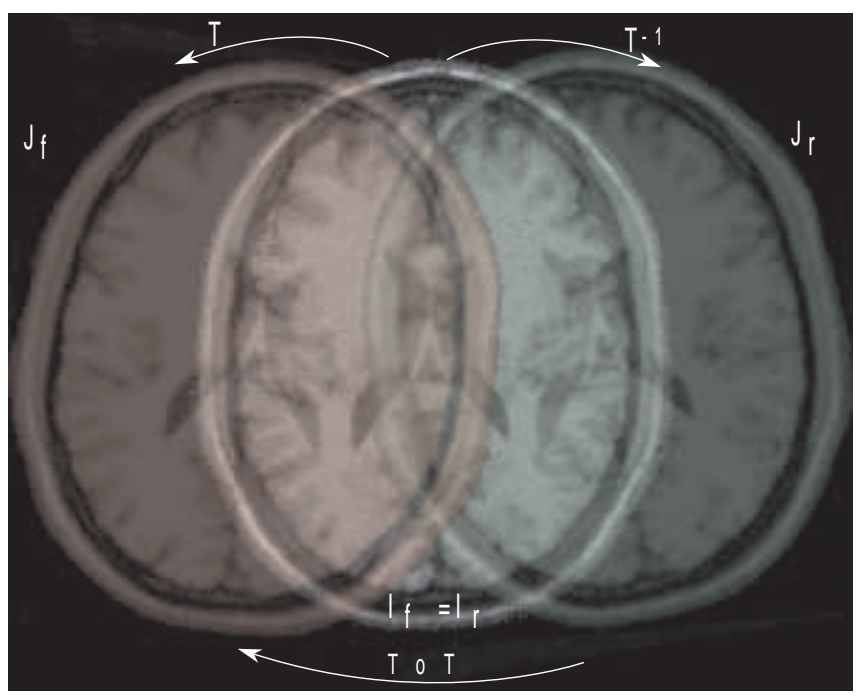

Figure 1. T1-to-T1 registration experiment. $I_{f}$ is the floating image and $I_{r}$ the reference image, with a perfect alignment. Two deformed images $I_{T}$ and $J_{T}$ are built following: $I_{T}(x)=I(T(x))$ and $J_{T}(x)=I\left(T^{-1}(x)\right)$, where $T$ is a random rigid transformation. As suggested in Thevenaz et al. ${ }^{11}$ both images are transformed, to lessen any bias introduced by interpolation artifacts since similar artifacts are introduced in both images. The registration process shall recover $R=T \circ T$ which aligns $I_{T}$ with $J_{T}$.

\subsection{Results}

Registration of the randomly transformed BrainWeb images (T1, T2 and PD) is performed for each combination of optimisation algorithms (NEWUOA, POW, SMP) and similarity measures (CC, MI, NMI, GMI). Figure 4 and Table 1 shows the accuracy of each similarity measure for each algorithm.

A paired Student's $t$-test was performed on the T2-to-T1 registration for each similarity measure, showing that NEWUOA has better accuracy $\left(p<1 e^{-4}\right)$ when compared to SMP and POW for MI, NMI and GMI. Keeping only the results from NEWUOA, the maximisation of MI leads to more accurate than the maximisation of both NMI $\left(p<1 e^{-4}\right)$ and GMI $\left(p<1 e^{-2}\right)$. Similar results were obtained for T1-to-T1 and T1-to-PD registration experiments. These results suggest that maximising MI with NEWUOA yields the best results in terms of accuracy in case of a single resolution scheme.

Further tests are made to show the impact of multiresolution strategies for the maximisation of MI by NEWUOA, SMP and POW. The comparison of the different algorithms for the MI is shown in Figure 3. There 


\begin{tabular}{|l|c|c|c||c|c|r|}
\hline & \multicolumn{3}{|c||}{ T1-to-T1 (mono-modal) } & \multicolumn{3}{c|}{ T2-to-T1 (multi-modal) } \\
\hline & robust. & acc. $(\mathrm{mm})$ & speed $(\mathrm{s})$ & robust. & acc. $(\mathrm{mm})$ & speed $(\mathrm{s})$ \\
\hline \hline SMP & $50 / 50$ & $0.16 \pm 0.02$ & $638 \pm 106$ & $50 / 50$ & $0.26 \pm 0.02$ & $688 \pm 10$ \\
\hline POW & $49 / 50$ & $0.11 \pm 0.00$ & $1809 \pm 330$ & $49 / 50$ & $0.16 \pm 0.00$ & $1900 \pm 51$ \\
\hline NEW & $50 / 50$ & $0.10 \pm 0.00$ & $453 \pm 38$ & $50 / 50$ & $0.13 \pm 0.00$ & $519 \pm 5$ \\
\hline \multicolumn{6}{c}{} & \multicolumn{4}{c|}{ PD-to-T1 (multi-modal) } \\
\cline { 2 - 6 } & & robust. & acc. $(\mathrm{mm})$ & speed $(\mathrm{s})$ \\
\cline { 2 - 6 } & & SMP & $49 / 50$ & $0.25 \pm 0.01$ & $433 \pm 72$ \\
& POW & $50 / 50$ & $0.13 \pm 0.00$ & $1123 \pm 224$ \\
& NEW & $49 / 50$ & $0.12 \pm 0.00$ & $307 \pm 28$ \\
\cline { 3 - 6 }
\end{tabular}

Table 1. Robustness, Accuracy and Speed results for MI, for each optimisation method studied in this paper with a mono resolution scheme. Results are obtained from 50 randomly generated transformations as described in 3.2. The robustness is the number of trials reaching a sub-voxel accuracy.

is no significant change in accuracy using these multiresolution strategies. Figure 5 shows a comparison of the run times for each pair of optimisation methods and similarity measures. NEWUOA has a clear advantage in terms of speed compared to the other algorithms, for any similarity measure. Figure 2 shows the impact of multiresolution schemes on the run time of each algorithm for the MI. With and without multiresolution NEWUOA is clearly faster than POW, and has a little advantage over SMP. For T1-to-T1 using MI without multiresolution execution time shows that NEWUOA is the fastest method compared to POW $\left(p<10^{-23}\right)$ and SMP $\left(p<10^{-13}\right)$, POW being slower than SMP $\left(p<10^{-21}\right)$. These results are in line with the literature. ${ }^{12}$ The optimisation time in Figure 5 shows that NEWUOA is faster than POW and SMP, and this holds for our multiresolution schemes.

The algorithms are considered successful when the warping index $\omega$ is less than $1 \mathrm{~mm}$ (subvoxel accuracy). NEWUOA is the algorithm that fails less often, followed by SMP. 

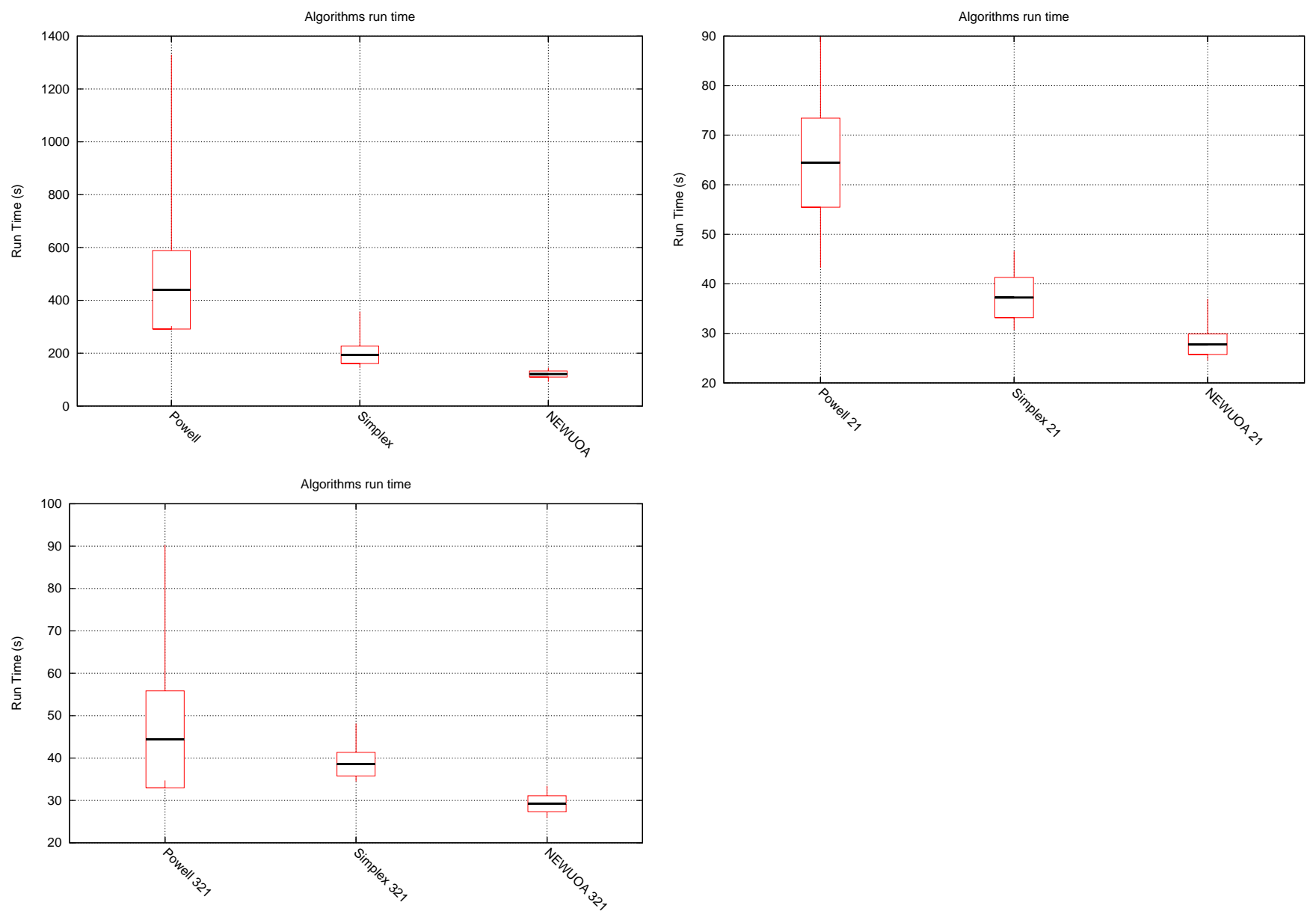

Figure 2. Comparison of the three different algorithms using the Mutual Information as a similarity measure for T1 to T1 registration and several multiresolution schemes. 1) at full resolution (Powell, Simplex, Newuoa), 2) at a resolution of half the original followed by a full resolution step (suffix 21), 3) at a resolution of a third the original followed by a resolution of half the original resolution and a final step at full resolution (suffix 321). Run time comparison using several multiresolution schemes. 


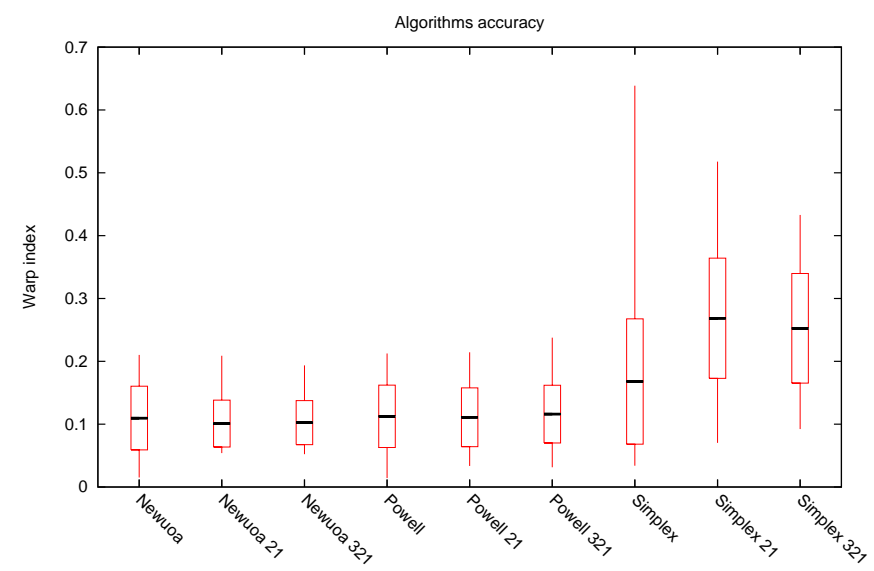

Figure 3. Comparison of the three different algorithms using the Mutual Information as a similarity measure for T1 to $\mathrm{T} 1$ registration and several multiresolution schemes. 1) at full resolution (Powell, Simplex, Newuoa), 2) at a resolution of half the original followed by a full resolution step (suffix 21), 3) at a resolution of a third the original followed by a resolution of half the original resolution and a final step at full resolution (suffix 321). Registration error measured in terms of warping index measure.
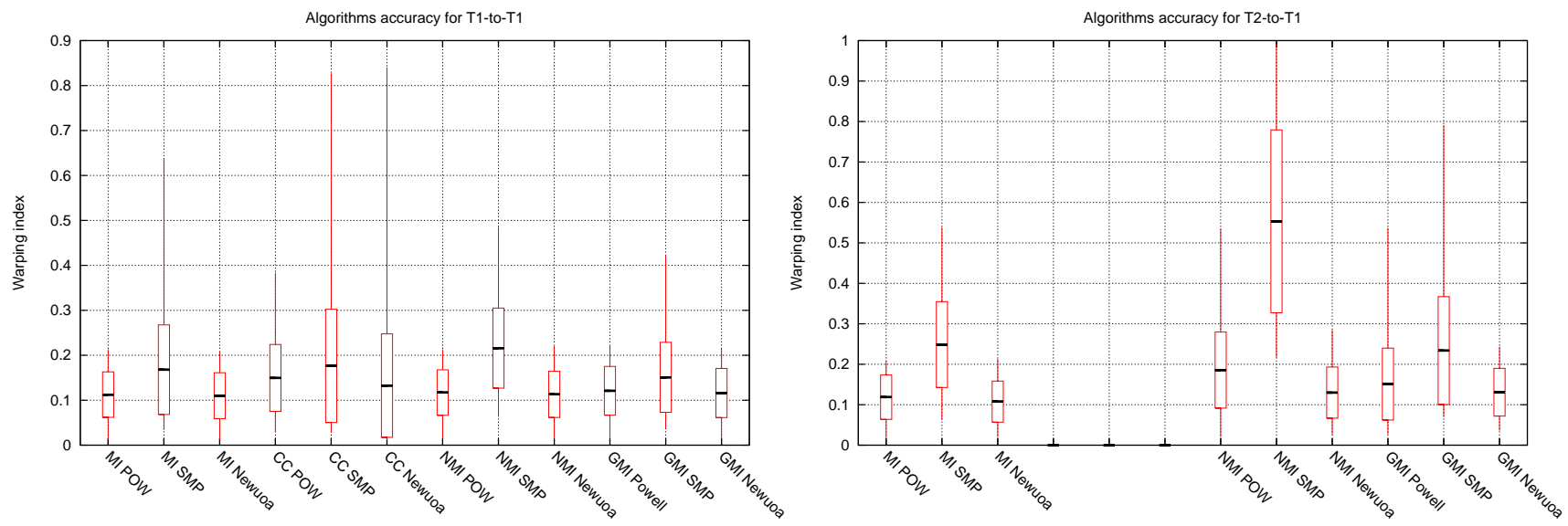

Figure 4. Comparison of the accuracy of the three algorithms Powell (POW), Simplex (SMP) and Newuoa. Left: T1-to$\mathrm{T} 1$ registration with the four different similarity measures (MI, CC, NMI, GMI). Right: T1-to-T2 registration with the three multi-modal similarity measures (MI, NMI, GMI). The correlation coefficient has been omitted for the T1-to-T2 registration as it is not suited for multi-modal registration. 

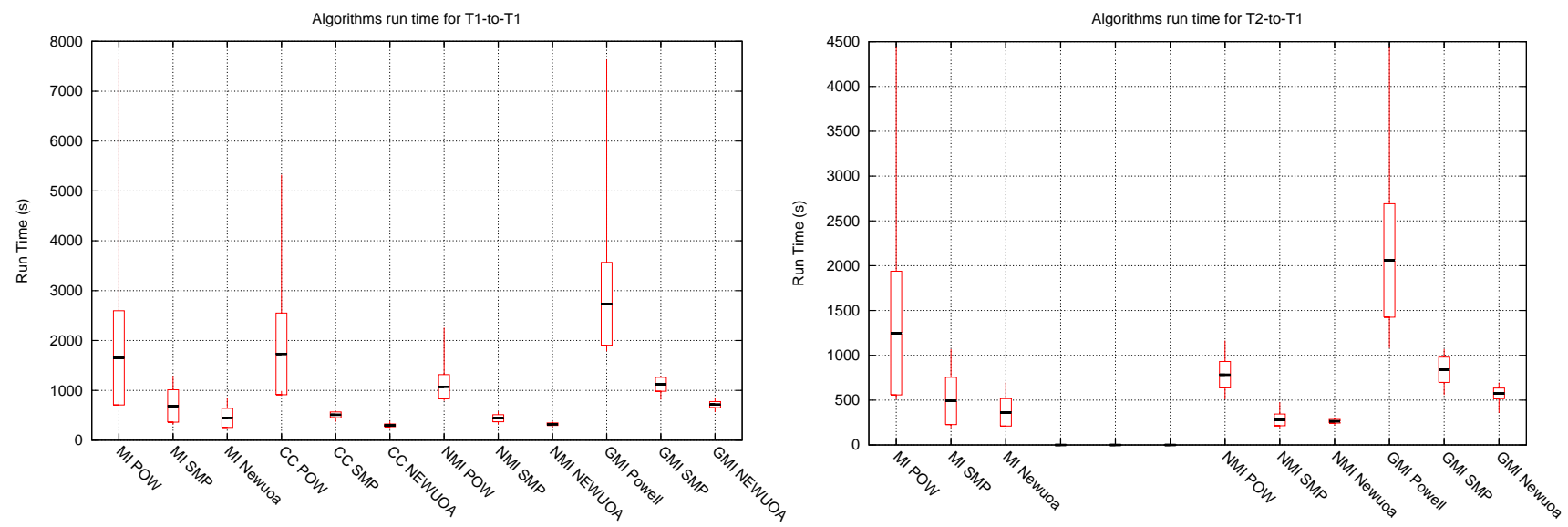

Figure 5. Comparison of the run time of the three algorithms Powell (POW), Simplex (SMP) and Newuoa. Left: T1to-T1 registration with the four different similarity measures (MI, CC, NMI, GMI). Right: T1-to-T2 registration with the three multi-modal similarity measures (MI, NMI, GMI). The correlation coefficient has been omited for the T1-to-T2 registration as it is not suited for multimodal registration.

\section{DISCUSSION}

This comparison of different similarity measures and optimisation algorithms differs from previous works on the same topic. For instance, West et al. ${ }^{13}$ compare different registration methods on a global point of view: no conclusion can be drawn about why a given method outperforms another one, as the methods under study often differ in many implementation choices (estimation of the joint densities, interpolations schemes, multiresolution strategies, etc.). On the opposite, our approach allows the focused comparison of key points of intensity based registration methods (i.e. similarity measures and optimisation algorithms) in a fair manner, as the other parameters of the implementation are held constant. Another interesting validation method has been led by Skerl et al.,${ }^{14}$ who focused on similarity measures based on probing curves, in an optimisation-free context.

\section{CONCLUSION}

A new optimisation method, NEWUOA, was proposed and evaluated for mono- and multi-modal rigid registration procedures. This method shows better robustness, accuracy and speed compared to the widely used Powell's direction set (POW) and Nelder-Mead's downhill simplex (SMP). The comparison of similarity criteria, performed in a fair manner, due to a common validation framework, shows which measure is best suited for a given registration problem regarding the optimisation scheme. As a conclusion, Mutual Information is a good trade-off for accuracy and speed, independently of the optimisation method. The comparison shows that NEWUOA is better suited for maximisation of MI for rigid registration purposes than POW and SMP. Finally, results on real data are shown for other modalities (PET-to-MRI and SPECT-to-MRI registration), obtained by maximisation of mutual information with NEWUOA (see Fig. 6).

\section{Acknowledgements}

Nicolas Wiest-Daesslé is supported by a grant from the ARSEP (French association for research on multiple sclerosis - Association pour la Recherche sur la Sclérose en Plaques). We thank Sean P. Morrissey for review of the manuscript and for his constant encouragements.

\section{REFERENCES}

1. J. Maintz and M. Viergever, "A survey of medical image registration," Medical Image Analysis 2, pp. 1-36, Mar. 1998. 


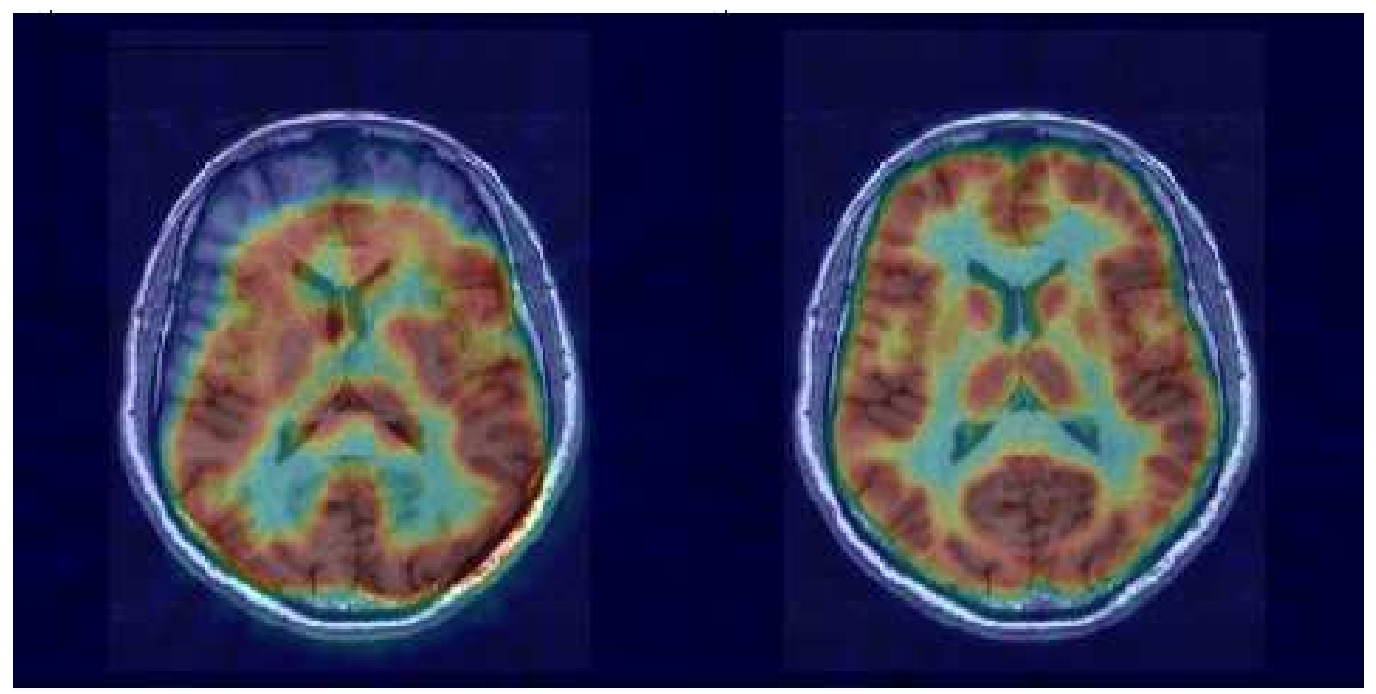

a

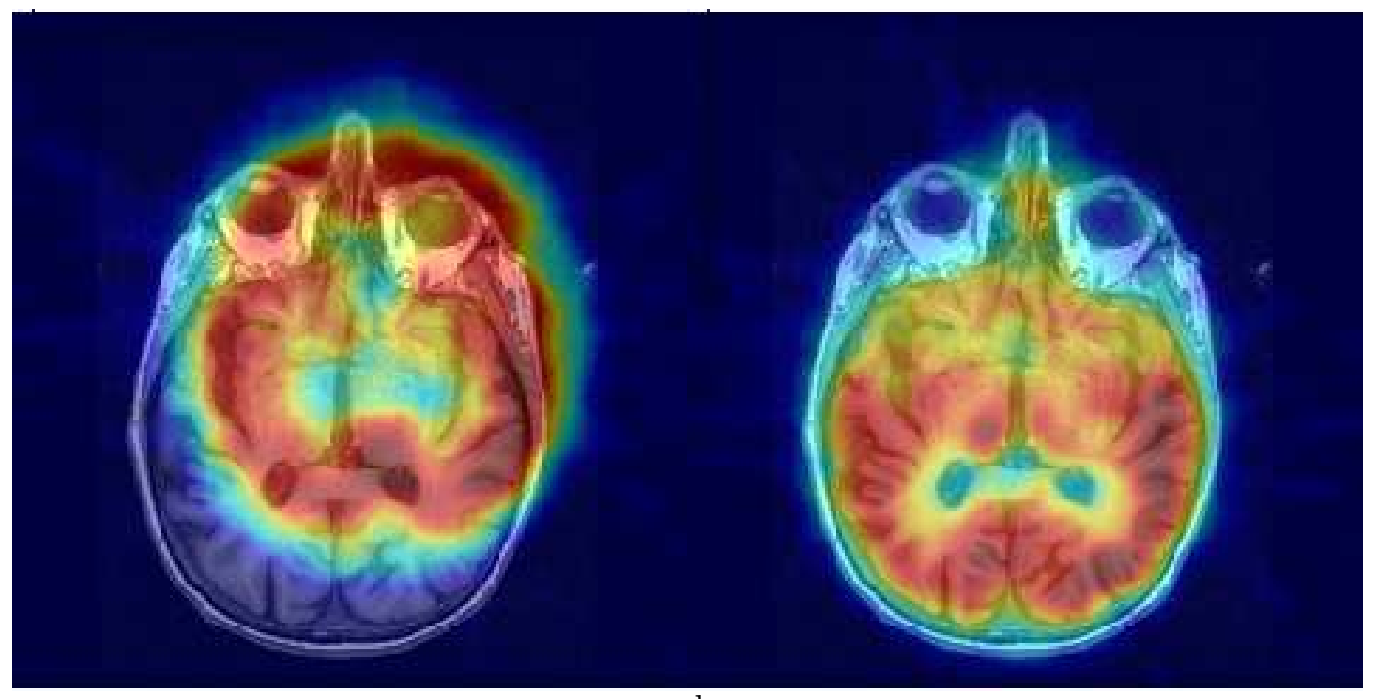

$\mathrm{b}$

Figure 6. Registration results on real data. a) PET-to-MRI registration. b) SPECT-to-MRI registration. In a) and b) original images are displayed at left and registered images at right. The floating image is represented overlaid on the reference image with a blue-red colormap.

2. M. Powell, "The NEWUOA Software for Unconstrained Optimization without Derivatives," in Workshop On Large Scale Nonlinear Optimization, G. Di Pillo and M. Roma, eds., Nonconvex Optimization and Its Applications 83, Springer, (Erice, Italy), July 2004.

3. W. H. Press, B. P. Flannery, S. A. Teukolsky, and W. T. Vetterling, Numerical Recipes: The Art of Scientific Computing, Cambridge University Press, Cambridge (UK) and New York, 2nd ed., 1992.

4. D. L. Collins, A. P. Zijdenbos, V. Kollokian, J. G. Sled, N. J. Kabani, C. J. Holmes, and A. C. Evans, "Design and construction of a realistic digital brain phantom.," IEEE Trans. Med. Imaging 17(3), pp. 463-468, 1998.

5. M. Powell, "UOBYQA: unconstrained optimization by quadratic approximation," tech. rep., Department of Applied Mathematics and Theoretical Physics, University of Cambdrige, 2000.

6. D. Winfield, "Function minimisation by interpolation in a data table," J. Inst. Maths Applics , 1973. 
7. A. Roche, G. Malandain, and N. Ayache, "Unifying maximum likelihood approaches in medical image registration," Research Report RR-3741, INRIA, Jul 1999.

8. F. Maes, A. Collignon, D. Vandermeulen, G. Marchal, and P. Suetens, "Multimodality image registration by maximization of mutual information," IEEE Transactions on Medical Imaging 16, pp. 187-198, Apr. 1997.

9. Z. Knops, J. Maintz, M. Viergever, and J. Pluim, "Normalized mutual information based PET-MR registration using K-Means clustering and shading correction," in Second International Workshop on Biomedical Image Registration, WBIR'2003, J. Gee, J. Maintz, and M. Vannier, eds., Lecture Notes in Computer Science 2717, pp. 31-39, Springer, (Philadelphia, USA), June 2003.

10. J. P. Pluim, J. B. Maintz, and M. A. Viergever, "Image registration by maximization of combined mutual information and gradient information.," IEEE Trans Med Imaging 19, pp. 809-814, August 2000.

11. P. Thévenaz and M. Unser, "Optimization of mutual information for multiresolution image registration," IEEE Transactions on Image Processing 9, pp. 2083-2099, Dec. 2000.

12. F. Maes, D. Vandermeulen, and P. Suetens, "Comparative evaluation of multiresolution optimization strategies for multimodality image registration by maximization of mutual information," Medical Image Analysis $\mathbf{3}$, pp. 373-386, Dec. 1999.

13. J. West, J. Fitzpatrick, M. Wang, B. Dawant, C. Maurer, R. Kessler, R. Maciunas, C. Barillot, D. Lemoine, A. Collignon, F. Maes, P. Suetens, D. Vandermeulen, P. Van Den Elsen, S. Napel, T. Sumanaweera, B. Harkness, P. Hemler, D. Hill, D. Hawkes, C. Studholme, J. Maintz, M. Viergever, G. Malandain, X. Pennec, M. Noz, G. Maguire, M. Pollack, C. Pellizzari, R. Robb, D. Hanson, and R. Woods, "Comparison and evaluation of retrospective intermodality brain image registration techniques," Journal of Computer Assisted Tomography 21, pp. 554-566, Jul-Aug 1997.

14. D. Skerl, B. Likar, and F. Pernus, "A protocol for evaluation of similarity measures for rigid registration," IEEE Transactions on Medical Imaging 25, pp. 779-791, June 2006. 